\title{
Toxicity of Acrylamide Derivatives to Embryos of the Rotifer Adineta vaga
}

\author{
Aydin Örstan \\ Institute of Gerontology, University of Michigan, 300 N. Ingalls, \\ Ann Arbor, Michigan 48109, USA
}

The invertebrate rotifer, introduced into toxicological research more than 40 years ago (Marriott et al. 1948; Allee and Rosenthal 1949), has since been used both as a model organism to investigate the biochemical and physiological actions of toxins (Lindner and Goldman 1964; Kleinow 1986) and as a test organism in bioassays to monitor pollution in marine and fresh water environments (Snell and Persoone 1989; Fernández-Casalderrey et al. 1991). The selection of rotifers as the experimental animals has several justifications. First, being multicellular animals, they offer a better choice over bacteria and protozoans. Second, they are easy to collect, culture and inexpensive to maintain. Third, several species are all parthenogenetic females. This enables one to prepare clones of genetically identical individuals, all descendants of one female. Consequently, the genetic factor is largely eliminated from the interpretation of the experimental results. Fourth, since the lifespan of most species is short ( 5 to $40 \mathrm{~d}$ ), the chronic effects of toxins on lifespan and reproduction can be evaluated relatively quickly.

To further demonstrate the usefulness of rotifers in toxicological research, it would be helpful to perform comparative investigations using toxins known to be active both in rotifers and vertebrates. One candidate toxin for such studies is acrylamide, whose toxicity has been extensively investigated in a variety of vertebrate species ranging from goldfish to humans (Miller and Spencer 1985). Kleinow (1986) demonstrated the acute toxicity of acrylamide to the marine rotifer Brachionus plicatilis. I have studied the cytotoxic effects of acrylamide and two of its derivatives on the embryos of the common fresh water rotifer Adineta vaga. Results reported here indicate that the order of decreasing cytotoxicity is N,N'methylene-bis-acrylamide, acrylamide and methacrylamide. This sequence is identical to the relative cytotoxicities of these compounds to cultured neuronal cells from embryonic rat brains (Hayashi et al. 1989).

\section{MATERIALS AND METHODS}

Adineta vaga Davis 1873 , a cosmopolitan rotifer, is the most common member of its genus. It usually lives in mosses, lichens and puddles of rainwater. I originally isolated $A$. vaga from a sample of rainwater collected from a gutter containing decomposed plant material. The species identification is based on Donner's keys (1965). An initial clone, prepared with the offspring of an isolated individual, formed the basis of all subsequent cultures. No other rotifer species or protozoans

Send reprint requests to the author at 2016 Baltimore Rd., \# I-22, Rockville, MD 20851, USA. 
are present in the cultures. The culture medium is prepared by boiling 5 rice grains in $50 \mathrm{~mL}$ distilled water. Before use, the medium is filtered through a filter paper (Whatman \#541) and diluted two-fold with distilled water. Since these animals feed on particulate organic matter as well as bacteria (Ricci 1984), the medium is not sterilized. Ricci (1984) succesfully cultured $A$. vaga and other bdelloid rotifers in media based on deionized water and justified the use of deionized water by the fact that rainwater is commonly the only kind of water in the usual habitats of these species. Furthermore, by avoiding buffers and other salts, the possible adverse effects of these compounds on the reproduction and longevity of rotifers are eliminated (Lansing 1942). General information about $A$. vaga and related species is available in various sources (Hyman 1951; Donner 1966; Pennak 1989).

Although the mothers' ages seem to have no effect on the hatchability of eggs, all the eggs used in the experiments were obtained from mothers not older than approximately $7 \mathrm{~d}$. Eggs were collected once every 20 to $24 \mathrm{hr}$. Eggs older than $24 \mathrm{hr}$ were not used. Eggs were incubated in depression slides in solutions of the compounds being tested. At least 26 eggs were used for each concentration of a test compound. Incubations were in the dark at room temperature $\left(21-23^{\circ} \mathrm{C}\right)$. To slow down evaporation, depression slides were kept in sealed plastic boxes. The number of eggs hatched were counted once a day for 5 to $6 \mathrm{~d}$ before the remaining eggs were discarded. Acute toxicity tests were performed by incubating groups of 10 to 20 animals of various ages with the compounds being tested. During acute toxicity tests the rotifers were not fed.

The solutions of acrylamide (Sigma Chemical Co., St. Louis, Missouri), methacrylamide (Aldrich Chemical Co., Milwaukee, Wisconsin) and N,N'methylene-bis-acrylamide (International Biotechnologies, Inc., New Haven, Connecticut) were prepared in distilled water without further purification.

\section{RESULTS AND DISCUSSION}

Fully grown animals from the clone of $A$. vaga used in this work are approximately $400 \mu \mathrm{m}$ long. Spurs are $6 \mu \mathrm{m}$ long. Reproduction is solely by parthenogenesis. In boiled rice medium, the longest life span recorded was about $39 \mathrm{~d}$ and the maximum number of eggs laid by one rotifer was 27. Animals usually lay eggs between the ages of 2 to $16 \mathrm{~d}$.

Under normal conditions, $A$. vaga will occasionally spend some time in a half contracted state with the toes anchored to a substrate and head pulled into the trunk. Under conditions of stress, such as during desiccation, in the absence of food or in the presence of toxins in the culture medium, both the head and the foot are withdrawn into the trunk which assumes an almost spherical shape. Older animals also spend most of their time in a contracted state. Thus, the observation of a large number of contracted animals in a culture of young rotifers almost always signals the presence of an adverse condition, usually a toxin. The contracted animals may remain alive in this state for several days. In the contracted form the organs are tightly packed into the trunk leaving no empty spaces. The death of a contracted $A$. vaga is indicated by gross morphological changes: the feet and the head extend out and become distorted, the organs begin to disintegrate and consequently, large gaps appear among the remaining tissues. During the acute toxicity tests I have differentiated between live and dead animals based on these criteria. However, since the number of eggs hatching within a given time period is a less ambiguous criterion than the somewhat arbitrary morphological changes listed above, most of 


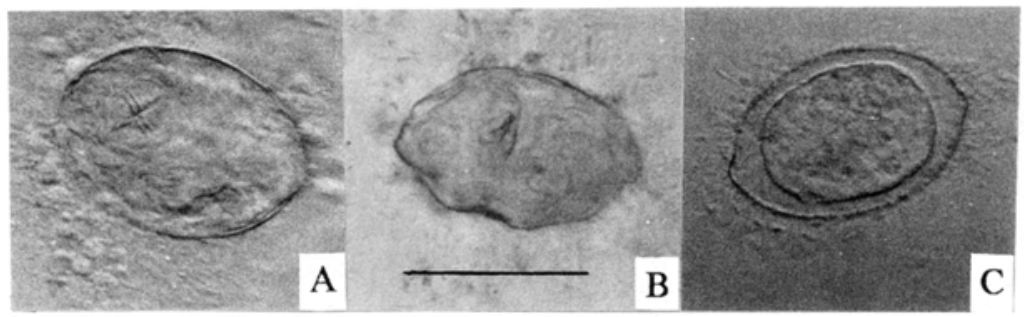

Figure 1. A: Live embryo of $A$. vaga incubated for approximately $48 \mathrm{hr}$ in distilled water. B: Live embryo of $A$. vaga incubated for approximately $50 \mathrm{hr}$ in $0.1 \mathrm{M}$ sucrose. Trophi are visible in A and B. C: The shrunken embryo in an egg incubated in $0.01 \mathrm{M}$ acrylamide for $43 \mathrm{hr}$. Scale bar is $50 \mu \mathrm{m}$.

the experiments reported here have been done with embryos instead of adult animals.

The eggs of $A$. vaga are ellipsoid with smooth shells (Figure 1). The average dimensions of the eggs are $67 \times 43 \mu \mathrm{m}$. The egg shell, which is less than $0.5 \mu \mathrm{m}$ thick, is readily permeable to water; shriveled dry eggs assume their normal shape within a minute after the addition of water. The eggs deform in $0.1 \mathrm{M}$ sucrose (Figure 1B) but retain their shape in $0.1 \mathrm{M}$ methacrylamide, indicating that the shell is not permeable to sucrose but permeable to methacrylamide. I have followed the initial development of several embryos incubated in distilled water at $21-23^{\circ} \mathrm{C}$. The first cleavage is completed in about an hour following the oviposition. At the end of the first $2 \mathrm{hr}$, embryos contain eight cells. Towards the end of the first $24 \mathrm{hr}$, the trophi become visible for the first time in unstained embryos examined at $400 \mathrm{X}$. At about the same time, the embryo begins to move inside the egg. The eggs hatch in about $48-55 \mathrm{hr}$ (Figure 1A).

During the course of this work, a total of 480 eggs of $A$. vaga were incubated either in distilled water or in boiled rice medium without any other additive. All except five of these eggs hatched. Four of the eggs that didn't hatch were kept under unsupported cover glasses. If these four eggs are excluded from the total, the percentage that hatched is 99.8 .

To investigate the acrylamide intoxication of embryos as a function of time, groups of eggs, collected within $24 \mathrm{hr}$ of oviposition, were first incubated in $0.01 \mathrm{M}$ acrylamide for various periods ranging from 4.3 to $20 \mathrm{hr}$. At the end of the incubation periods acrylamide solutions were replaced with distilled water and the number of eggs that hatched in the following $6 \mathrm{~d}$ were determined. The results in Table 1 indicate that $12 \%$ of the embryos survive an incubation period of $20 \mathrm{hr}$ in $0.01 \mathrm{M}$ acrylamide. To obtain comparative data, acute toxicity of acrylamide in adult rotifers was evaluated. In a group of 32 adult rotifers placed in $0.01 \mathrm{M}$ acrylamide, $11(34 \%)$ remained active for about $35 \mathrm{hr}$. This suggests that the embryos may be more sensitive to acrylamide than adults. The first deaths in the adult population kept in acrylamide were recorded after a period of about $72 \mathrm{hr}$. At the end of the fifth day all the rotifers were dead. Whereas in a control population of 27 rotifers kept in distilled water, on the fifth day $19(70 \%)$ of the rotifers were still active and only one rotifer was dead, indicating that the lack of food was not the main factor responsible for the early deaths recorded in the acrylamide population. 
Table 1. Percentage of $A$. vaga eggs hatched after incubation in $0.01 \mathrm{M}$ acrylamide for the indicated time periods. Following exposure to acrylamide, eggs were kept in distilled water. At least 26 eggs, collected within $24 \mathrm{hr}$ of oviposition, were used for each group.

Incubation time (hr) \% Hatched

$\begin{array}{cc}4.3 & 59 \\ 6 & 42 \\ 8 & 41 \\ 10 & 20 \\ 16 & 13 \\ 20 & 12\end{array}$

Increased sensitivities of embryos to acrylamide may have several causes. For example, the detoxification mechanisms that normally operate in the cells of adult rotifers may not be fully developed in embryos. Acrylamide reversibly or irreversibly inhibits several enzymes (Örstan and Gafni 1990). Thus, it is also possible that certain enzymes that are expressed only in the embryonic rotifer cells may be highly susceptible to inactivation by acrylamide. The inactivation of these enzymes by acrylamide may consequently block cell division or kill the cells.

Contrary to the development of the normal embryos, some embryos incubated in the presence of acrylamide or one its derivatives take on a strikingly different appearance. The embryo coagulates and shrinks away from the surrounding shell (Figure 1C) and never hatches. Since easily identifiable structures, such as trophi, cannot be observed in these embryos, this type of embryonic death appears to result from the cytotoxic action of acrylamide on most or all of the embryonic cells during the early stages of development. On the other hand, some embryos, especially at low concentrations of toxins, develop apparently normally and resemble the control eggs but nevertheless fail to hatch. In these cases, the toxic action of acrylamide is probably directed on a specific target tissue, for example, the nervous system, of the embryos.

When the eggs were incubated for 5 to $6 \mathrm{~d}$ in the presence of the test compounds, the percentage of the eggs that hatched depended on the compound and its concentration (Figure 2). I have estimated the approximate concentrations required to kill $50 \%$ of the embryos from the curves in Figure 2. In terms of these concentrations, the most toxic compound is $\mathbf{N}, \mathbf{N}^{\prime}$-methylene-bis-acrylamide $(0.0012 \mathrm{M})$, followed by acrylamide $(0.0031 \mathrm{M})$ and methacrylamide $(0.04 \mathrm{M})$. This sequence is identical to that reported by Hayashi et al., (1989) for the cytotoxicity of these three compounds towards cultured neuronal cells from brains of rat embryos.

This sequence of cytotoxicity weakens the possibility that the cytotoxic effects are due to the changes in the osmolarity of the incubation medium. If osmolarity were the only factor responsible for the killing of the embryos, then at a given concentration the same percentage of eggs would hatch regardless of the toxin present. 


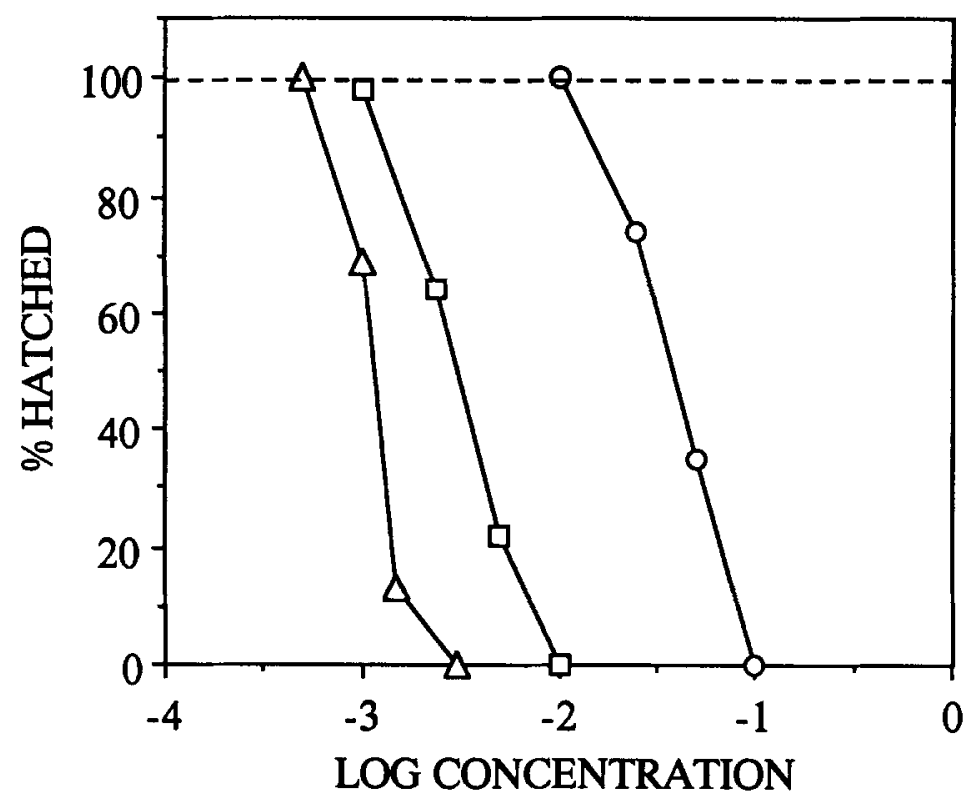

Figure 2. Concentration (M) dependent effects of $\mathrm{N}, \mathrm{N}^{\prime}$-methylene-bis-acrylamide $(\Delta)$, acrylamide ( $\square$ ) and methacrylamide (o) on the hatching of $A$. vaga eggs. At least 26 eggs, collected within $24 \mathrm{hr}$ of oviposition, were incubated at each concentration for 5 to $6 \mathrm{~d}$. The dotted line indicates the percentage of control eggs hatched $(99.8 \%)$. The curves connecting the points have no theoretical basis.

The direct effect of osmolarity on the hatching of eggs was also investigated in a set of experiments using sucrose to increase the osmolarity of the incubation media. Out of a group of 32 eggs incubated in $0.01 \mathrm{M}$ sucrose, 30 hatched. In $0.05 \mathrm{M}$ sucrose, 13 out of a total of 21 eggs hatched in $4 \mathrm{~d}$ and after the remaining eggs were transferred to distilled water, 7 more hatched. In $0.1 \mathrm{M}$ sucrose, none of the 20 eggs hatched in $4 \mathrm{~d}$. However, after 19 of these eggs were transferred to distilled water, 18 of them hatched. These results indicate that the majority of eggs survive for at least $4 \mathrm{~d}$ in $0.05-0.1 \mathrm{M}$ sucrose, whereas in $0.01 \mathrm{M}$ acrylamide, only $12 \%$ survive beyond $20 \mathrm{hr}$ (Table 1). These results again point out that osmolarity is not an important factor in the killing of eggs in solutions of acrylamide or its derivatives. Furthermore, in the presence of high concentrations of sucrose, both the embryo and the egg shell deform, although the embryo continues to develop (Figure 1B). This is clearly different than the results obtained with acrylamide, in which case, as already discussed, the embryo shrinks and stops developing but the egg shell retains its normal shape (Figure 1C).

Despite its cytotoxicity, $\mathbf{N}, \mathbf{N}^{\prime}$-methylene-bis-acrylamide is not neurotoxic in mice (Hashimoto et al., 1981). Presumably, two different biochemical mechanisms are responsible for the cytotoxic and the neurotoxic actions of acrylamide and its derivatives. Since the relative cytotoxicities of N,N'-methylene-bis-acrylamide, acrylamide and methacrylamide are identical in rotifers and rat neuronal cells, the mechanisms of cytotoxicity of these compounds may also be identical in vertebrate and invertebrate species. 
These results support the use of rotifers in toxicity tests and bioassays. The information from rotifer based tests would be valuable in the evaluation of the effects of toxins and environmental contaminants in vertebrates.

Acknowledgments. I thank Dr. A. Gafni for his support and interest in this study and Dr. K. Flurkey for a critical review of the manuscript.

\section{REFERENCES}

Allee WC, Rosenthal GM (1949) Group survival value for Philodina roseola, a rotifer. Ecology 30:395-397

Donner J (1965) Ordnung Bdelloidea. Akademie-Verlag, Berlin

Donner J (1966) Rotifers. Frederick Warne, London

Fernández-Casalderrey A, Ferrando, MD, Gamón, M, Andreu-Moliner, E (1991)

Acute toxicity and bioaccumulation of endosulfan in rotifer (Brachionus

calyciflorus) Comp Biochem Physiol 100C:61-63

Hashimoto K, Sakamoto J, Tanii H (1981) Neurotoxicity of acrylamide and related compounds and their effects on male gonads in mice. Arch Toxicol 47:179-189

Hayashi M, Tanii H, Horiguchi M, Hashimoto K (1989) Cytotoxic effects of acrylamide and its related compounds assessed by protein content, LDH activity and cumulative glucose consumption of neuron-rich cultures in a chemically defined medium. Arch Toxicol 63:308-313

Hyman LH (1951) The invertebrates, vol 3. McGraw-Hill, New York, pp 59151

Kleinow W (1986) Effects of acrylamide on Brachionus plicatilis (Rotifera). Comp Biochem Physiol 84C:243-246

Lansing AI (1942) Some effects of hydrogen ion concentration, total salt concentration, calcium and citrate on longevity and fecundity of the rotifer. J Exp Zool 91:195-211

Lindner LA, Goldman, H (1964) The influence of cholinergic and anticholinergic drugs on egg deposition by a rotifer. J Pharmacol Exp Therap 146:123-128

Marriott RH, Morris S, Larthe Y (1948) Use of rotifera for testing analgesics, narcotics and similar drugs. Nature 162:189-190

Miller MS, Spencer PS (1985) The mechanisms of acrylamide axonopathy. Ann Rev Pharmacol Toxicol 25:643-666

Örstan A, Gafni A (1990) The interaction of acrylamide with glyceraldehyde-3phosphate dehydrogenase. Structural modifications in the enzyme studied by fluorescence techniques. Photochem Photobiol 51:725-731

Pennak RW (1989) Fresh-water invertebrates of the United States. John Wiley, New York, pp 169-225

Ricci C (1984) Culturing of some bdelloid rotifers. Hydrobiol 112:45-51

Snell TW, Persoone G (1989) Acute toxicity bioassays using rotifers. II. A freshwater test with Brachionus rubens. Aquat Toxicol 14:81-92 